Haben Sie Fragen zur Abrechnung oder zur wirtschaftlichen Experten wenden: Helmut Walbert, Facharzt für Allgemeinmedizin und Betriebswirt aus Würzburg.

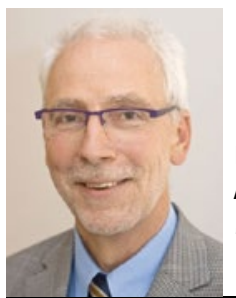

Helmut Walbert Allgemeinarzt, Medizinjournalist und Betriebswirt Medizin

\title{
Routinekontrollen
}

\section{Heimleitung will Rezepte für BZ-Teststreifen}

\section{$?$ Dr. H. G. Allgemeinarzt, Bayern: Ein Altenheim kontrolliert routi- nemäßig bei Diabetikern die Blutzu- ckerwerte. Jetzt möchte die Heimlei-}

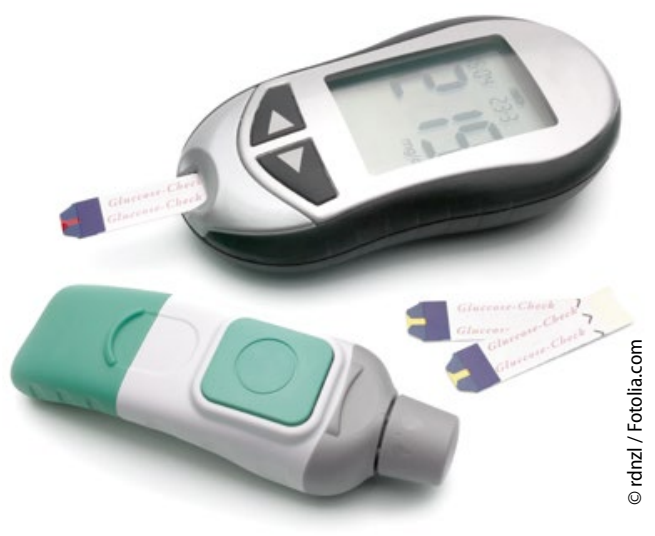

\section{tung für jeden Diabetiker einmal im Quartal die Verordnung von Blut- zuckerteststreifen. Geht das?}

!

MMW-Experte Walbert: Auch in Altenheimen gelten die gleichen Verordnungseinschränkungen wie sonst in der ambulanten Behandlung: Teststreifen werden nur bei Insulin spritzenden Diabetikern übernommen, außerdem vorübergehend bei einer Therapieumstellung oraler Antidiabetika, wenn eine Hypoglykämiegefahr droht.

Grundsätzlich sind solche „Routinen“ in Altenheimen kritisch zu sehen. Vom Messen wird kein Blutzucker besser! Für therapeutische Konsequenzen aufgrund der Messergebnisse fehlt in den Heimen nicht nur die Qualifikation des Personals, sondern auch die damit verbundene Entscheidungsverantwortung. Für die Akutdiagnostik in "Notfällen“ gehört ein Blutzuckermessgerät zu der vom Standardausrüstung, die das Heim vorhalten muss - inklusive Teststreifen. Hier gebe ich im Übrigen bei entsprechenden Anfragen in der Regel meine Zustimmung, das Messgerät eines meiner „Insuliner“ zu nutzen.

Sollten Angehörige die medizinisch nicht begründete Blutzucker-Routinekontrolle im Heim wünschen, kann selbstverständlich ein Privatrezept ausgestellt werden.

\section{Arbeitsrecht}

\section{Befristeter Vertrag für ehemalige Mitarbeiterin?}

?

Dr. M. W. Hausarzt-Internistin, Nordrhein: Ich möchte eine ehemalige Mitarbeiterin wieder einstellen, vorerst aber mit befristetem Arbeitsvertrag, um mich ggf. problemlos umentscheiden zu können. Geht das?

MMW-Experte Walbert: Die Nachfrage ist mehr als berechtigt: Befristete Arbeitsverträge für frühere Mitarbeiter gibt es nur noch mit vorliegendem
Sachgrund, der im Arbeitsvertrag genannt sein sollte. Häufigster Grund ist die Vertretung wegen Schwangerschaft, Mutterschaft, Elternzeit oder Krankheit einer anderen Mitarbeiterin.

Ohne solche Sachgründe setzt die Mitarbeiterin ihr früheres Arbeitsverhältnis unbefristet fort, wie das Landesarbeitsgericht Baden-Württemberg geurteilt hat. Es stellte mit Verweis auf $\$ 14$ Teilzeit- und Befristungsgesetz (TzBfG) fest, dass auch länger zurückliegende Beschäftigungen ohne Sachbegründung eine Befristung unmöglich machen (Az.: 6 Sa 28/13). In dem Paragrafen sind im Übrigen auch die anerkannten Sachgründe aufgezählt. Wollen Sie eine rechtlich einwandfreie Ausführung des Arbeitsvertrages sicherstellen, rate ich, einen Rechtsanwalt hinzu zu ziehen. Dieser haftet dann auch, wenn trotzdem etwas schiefgeht. 